\title{
Examination of HLA-DR4 as a severity marker for rheumatoid arthritis in Greek patients
}

\author{
Kyriaki A Boki, Alexandros A Drosos, Athanasios G Tzioufas, Jerry S Lanchbury, \\ Gabriel S Panayi, Haralampos M Moutsopoulos
}

\begin{abstract}
Objectives-Previous reports have shown that HLA-DR4 may be a severity marker for rheumatoid arthritis (RA) in patients of northern European origin. The aim of the present study was to investigate this relation in Greek patients with $R A$, as $R A$ in Greece differs from the $R A$ described previously on clinical, serological, and immunological grounds.
\end{abstract}

Methods-Eighty four patients were studied in whom HLA-DR typing was performed by restriction fragment length polymorphism and the subtypes of HLADR4 were determined by the polymerase chain reaction. The absence or presence of HLA-DR4 and its subtypes was correlated with the clinical and serological characteristics of the patients and with the side effects due to disease modifying drugs.

Results-Twenty one of the $84(25 \%)$ patients with RA were DR4+. There was no difference between the DR4+ and DR4patients with respect to duration of disease, severity of arthritis, functional grade, and joint erosion score. The DR4+ group were more likely to have side effects due to disease modifying drugs $(43 \%)$ than DR4- patients $(36 \%)$, but this difference was not statistically significant. DR4patients had more extra-articular manifestations, including Sjögren's syndrome (47 $v 19 \%$ ). Analysis of the DR4 subtypes showed that Dw15 was the most common variant $(9 / 21$ patients; $43 \%)$. There was no statistical difference in the clinical manifestations among patients with different DR4 subtypes. The same was also true when the clinical picture was correlated with the 'shared RA epitope' (QKRAA QRRAA/RRRAA), which is common to all HLA-DRB1 alleles positively associated with RA.

Conclusions-These results suggest that HLA-DR4 is not a severity marker in Greek patients with $R A$ and further indicate differences in the clinical expression of RA in Greece.

(Ann Rheum Dis 1993; 52: 517-519)

Several studies have shown that rheumatoid arthritis (RA) in Greece appears to differ on clinical, serological, and immunogenetic grounds from the RA found in patients of northern European origin. ${ }^{1}$ We carried out a prospective comparative study between British patients with RA at Guy's Hospital, London and Greek patients with RA from the department of internal medicine, Ioannina, performed by one observer. This study reported significant clinical differences in disease expression between the two patient groups, including more radiological erosive disease, poorer functional status, and a higher frequency of extra-articular manifestations in the British group. ${ }^{2}$ Furthermore, an immunogenetic study of the same Greek patients with RA revealed that only $43 \%$ had HLA-DR4 or the so called 'shared RA epitope', or both. ${ }^{3}$

These observations, coupled with previous reports in which DR4 has been proposed as a severity marker for $\mathrm{RA}^{45}$ prompted the present study, which attempts to address the question of whether DR4 is a severity marker of disease in Greek patients with RA.

\section{Subjects and methods}

PATIENTS

Eighty four unrelated adult Greek patients with $\mathrm{RA}$, selected according to the revised 1987 American Rheumatism Association (ARA) criteria, ${ }^{6}$ were studied. The patients were followed at the rheumatology outpatient clinic, department of internal medicine, University Hospital of Ioannina. From the patients' medical records the following data were collected: age, sex, disease duration, arthritis of small and large joints, extra-articular manifestations such as rheumatoid nodules, Raynaud's phenomenon, serositis (pleurisy or pericarditis), skin vasculitis, livedo reticularis, lymphadenopathy, and the presence of Sjögren's syndrome. Information on disease modifying drugs and their side effects (due to intramuscular gold salts or D-penicillamine) were also recorded. The following were considered as side effects: skin rashes, leucopenia, thrombocytopenia, proteinuria, myasthenia gravis, lupus-like syndrome, or pemphigus. The hand radiographs of all patients were evaluated using the Steinbrocker score. Additionally, the following laboratory variables were collected: packed cell volume, white blood cell count, platelet count, urine analysis, rheumatoid factor titre (latex fixation, positive titre $\geqslant 40$ ), antinuclear antibodies by indirect immunofluorescence using Hep-2 cell lines, and antibodies to extractable nuclear antigens by counterimmunoelectrophoresis. 
METHODS

$H L A$ class II typing by restriction fragment length polymorphism

DNA was extracted from blood samples by standard procedures. Restriction fragment length polymorphism for DRB was determined according to established methods. ${ }^{89}$

Polymerase chain reaction amplification and oligonucleotide hybridisation

Subtypes of HLA-DR4 were investigated at the nucleotide sequence level using polymerase chain reaction amplification as previously described. ${ }^{310}$ Subtypes of HLA-DR1 and the HLA-DRw6 complex were also examined by polymerase chain reaction amplification and oligonucleotide hybridisation as previously described. ${ }^{3} 11$

\section{Statistical analysis}

For statistical analysis Student's $t$ test and the $\chi^{2}$ test were used, with Yates's correction where necessary. Results are expressed as mean (SD) values.

\section{Results}

Table 1 summarises the clinical data of the two groups of patients with RA (DR4 positive and DR4 negative). The sex, mean age, mean disease duration, seropositivity, number of active joints, and incidence of antibodies to Ro (SSA) did not differ significantly between the two groups. When the extra-articular manifestations and the presence of Sjögren's syndrome together were analysed in the two groups of patients with RA, it was found that the DR4 negative patients presented this combination more often than DR4 positive patients $\left(47 \%\right.$ v $\left.19 \% ; \chi^{2}=4.21 ; \mathrm{p}<0.05\right)$. Changes in the hand radiographs did not differ significantly between the two groups of patients examined (table 2). The DR4 positive patients

Table 1 Rheumatoid arthritis in HLA-DR4 positive and HLA-DR4 negative Greek patients

\begin{tabular}{llll}
\hline Clinical findings & $\begin{array}{l}H L A-D R 4+ \\
(n=21)\end{array}$ & $\begin{array}{l}H L A-D R 4 \\
(n=63)\end{array}$ & $p$ Value \\
\hline Female/male & $17 / 4$ & $54 / 9$ & \\
Mean (SD) age (years) & $58 \cdot 2(12 \cdot 5)$ & $54 \cdot 1(11 \cdot 1)$ & NS \\
Mean (SD) disease duration (years) & $11 \cdot 0(8 \cdot 9)$ & $9 \cdot 6(6 \cdot 3)$ & NS \\
Seropositivity (\%) & 85 & 73 & NS \\
No with antibodies to Ro (SSA) & 4 & 7 & NS \\
Mean (SD) No of active joints & $5 \cdot 7(4 \cdot 2)$ & $6 \cdot 2(6 \cdot 5)$ & NS \\
No with extra-articular features & 1 & 12 & NS \\
No with Sjögren's syndrome & 3 & 18 & $<0 \cdot 05$ \\
No with EMSS & 4 & 30 & NS \\
No with side effects $\ddagger$ & 9 & 23 &
\end{tabular}

NS=not significant.

${ }^{\star}$ Rheumatoid nodules, Raynaud's phenomenon, serositis, skin vasculitis, livedo reticularis, and lymphadenopathy.

tExtra-articular features and Sjögren's syndrome.

$\ddagger$ Side effects due to sodium aurothiomalate or D-penicillamine (rash, leucopenia, thrombocytopenia, proteinuria).

Table 2 Hand radiographs scored according to the Steinbrocker classification in Greek patients with $R A$ who were $H L A-D R 4$ positive and $H L A-D R 4$ negative

\begin{tabular}{lll}
\hline Grade & Percentage of radiographs positive \\
\cline { 2 - 3 } & $H L A-D R 4+$ & $H L A-D R 4$ \\
\hline I & 38 & 29 \\
II & 38 & 41 \\
III & 19 & 16 \\
IV & 5 & 14 \\
\hline
\end{tabular}

had more side effects due to disease modifying drugs $(43 \%)$ than the DR4 negative patients $(36 \%)$, but this difference was not statistically significant.

Dw15 was the most common variant and was observed in nine patients $(43 \%$ of DR4 positive patients). The other DR4 subtypes (Dw4, Dw14, Dw13) were also detected but the numbers were too small for statistical analysis. An interesting observation was that all the Dw4, Dw14, and Dw13 patients were seropositive for rheumatoid factor. There was no difference in clinical manifestations among patients with different DR4 subtypes. The Dw10 variant was not detected in our patients with RA. The HLA-DR $\beta 1$ chains of Dw4, Dw14, and Dw15 variants of DR4, DR1, and DRw10 share a similar third hypervariable region amino acid sequence (QKRAA QRRAA/RRRAA on the single letter amino acid code), which is called 'the shared epitope'. ${ }^{12}$ When the clinical data for the patients with 'the shared RA epitope' was compared with the rest of the patients with RA, no statistical difference was observed. The Dw16 variant of DRw6 has a similar sequence (QRRAA) but it was not detected in our patients. $^{3}$

\section{Discussion}

Rheumatoid arthritis can present with a wide clinical spectrum of expression from mild to severe articular disease with or without extraarticular manifestations. It is associated with HLA-DR4 or DR1 and molecular analysis of the third hypervariable region of these molecules has revealed a similar molecular epitope which has substantiated the 'shared epitope' hypothesis first proposed by Gregersen et al. ${ }^{12}$ The implications for the presentation of unknown rheumatoid antigens to $T$ cells is obvious. Since the discovery of the association of RA with HLA-DR4, ${ }^{13}{ }^{14}$ various workers have attempted to define prognostic markers based on the presence or absence of HLA-DR4; some workers have suggested that patients with RA who are HLA-DR4 positive have more severe articular disease associated with extra-articular manifestations, ${ }^{5}{ }^{15-17}$ so that, for example, $96-100 \%$ of patients with RA and Felty's syndrome are DR4 positive, ${ }^{18-20}$ whereas other workers have not found these associations. $^{21} 22$

Rheumatoid arthritis in Greece is a milder joint disorder than RA in England, ${ }^{2}$ with significantly fewer extra-articular manifestations and an almost total absence of Felty's syndrome. ${ }^{2}$ These clinical differences may be partly based on immunogenetic differences as previously described by serological typing ${ }^{23}$ and extended by the present molecular analysis in which $43 \%$ of Greek patients with RA were shown to possess the disease susceptibility hypervariable region compared with British patients in whom it was present in over $80 \% .^{324}$ In the present study, evaluation of the articular and extra-articular manifestations of Greek patients with RA with or without HLADR4 or its subtypes did not reveal any 
significant differences. The observation that Greek patients with RA with extra-articular manifestations belong to the DR4 negative group is due mainly to Sjögren's syndrome, as analysis of the extra-articular features of the patients excluding Sjögren's syndrome yielded no statistical differences between patients with RA with and without HLA-DR4 or its subtypes. Also of interest is the observation that all Greek patients with RA with the HLADR4 subtypes Dw4, Dw14, or Dw13 were seropositive for rheumatoid factor. Dw4 and Dw14 variants have previously been associated with seropositive RA. ${ }^{25}$ The mechanisms by which these DR4 Dw subtypes influence rheumatoid factor production is not known. Rheumatoid factor seropositivity in most studies, however, has been associated with severe erosive disease and the presence of extra-articular features, so the primary association is unclear. ${ }^{15-17}$

One way in which Greek patients with RA differ significantly from those of northern Europe is in the distribution of DR4 subtypes among DR4 positive patients. Rheumatoid arthritis and, particularly, Felty's syndrome, are most strongly associated with the HLADw4 subtype, which is much less common among DR4 positive Greek patients. ${ }^{3} 20$ A study of southern Chinese patients with RA has reported that although Dw14 and Dw15 were the most common subtypes among DR4 positive subjects, Dw4 was lacking and these patients showed relatively milder disease than that commonly encountered in northern Europe. ${ }^{26}$ This situation is similar to that in our Greek patients and may reflect the fact that the Dw4 subtype is the main determinant of disease severity in a population. Given its rarity in Greek patients, further large scale studies will be necessary to evaluate this hypothesis.

To explain the genetic heterogeneity among Greek patients with RA the 'shared RA epitope' was also investigated. It was found that only $43 \%$ of the patients had this epitope. This finding is in contrast with previous studies, where each of the major susceptibility genes associated with RA in DR4 positive and DR4 negative subjects share related amino acid sequences. ${ }^{25}$ Hence, this finding implies that the 'shared epitope' does not seem to play as crucial a part in the aetiopathogenesis of Greek patients with RA.

These observations further support the proposal that RA in Greek patients differs from RA reported previously mainly in patients of northern European origin. The clinical similarities of the Greek patients with RA with or without DR4 or its subtypes raise important questions about the role of these molecules in the initiation and perpetuation of autoimmune lesions of RA in Greece. One conclusion may be that DR4, DR1, and DR10 positivity, in this group, is a susceptibility rather than a severity marker. Furthermore, the immunogenetic and clinical features observed in Greek patients with RA suggest that other gene(s) in or outside the HLA region or environmental factors, or both, may be influencing or modifying disease expression.
We thank the Wellcome Trust for an inter-laboratory collaborative grant to JSL, GSP, and HMM and also the Arthritis and Rheumatism Council (grants U9 and P75, and L45).

1 Vlachoyiannopoulos P G, Moutsopoulos H M. Rheumatoid arthritis in southeastern Europe. Baillieres Clin Rheumatol 1992; 6: 221-3.

2 Drosos A A, Lanchbury J S, Panayi G S, Moutsopoulos H $M$. Rheumatoid arthritis in Greek and British patients: comparative clinical, radiological and serological study. Arthritis Rheum 1992; 35: 745-8.

3 Boki K A, Panayi G S, Vaughan R W, Drosos A A Moutsopoulos H M, Lanchbury J S S. HLA class I sequence polymorphisms and susceptibility to rheumatoid arthritis in Greece. Arthritis Rheum 1992; 35: 749-55.

4 Roitt I M, Corbett M, Festenstein H, et al. HLA-DRW4 and prognosis in rheumatoid arthritis [letter]. Lancet 1978; i: 990.

5 Young A, Jaraquemada D, Awad J, et al. Association of HLA-DR4/DW4 and DR2/DW2 with radiological changes in prospective study of patients with rheumatoid arthritis. Arthritis Rheum 1984; 27: 20-5.

6 Arnett F C, Edworthy S M, Bloch D A, et al. The American Rheumatism Association 1987 revised criteria for the classification of rheumatoid arthritis. Arthritis Rheum 1988; 31: 315-24.

7 Steinbrocker O, Trager C H, Batterman R C. Therapeutic criteria in rheumatoid arthritis and radiographic staging system for rheumatoid arthritis. $\mathcal{F} A M A$ 1949; 140: 659-62.

8 Bidwell J L, Jarrold E A. HLA-DR allogenotyping usin exon-specific CDNA probes and application of rapid exon-specific cDNA probes and application of
minigel methods. Mol Immunol 1986; 23: 1111-6.

9 Bidwell J L, Bidwell E A, Laundy G J, Klouda P T, Bradley $B$ A. Allogenotypes defined by short $D Q \alpha$ and $D Q \beta$ cDNA probes correlate with and define splits of HLA-DQ serological specificities. Mol Immunol 1987; 24: 513-22.

10 Lanchbury J S S, Hall M A, Welsh K I, Panayi G S Sequence analysis of HLA-DRB1 subtypes: additional first domain variability is detected by oligonucleotide hybridization and nucleotide sequencing. Hum Immunol 1990; 27: 136-44

11 Vaughan R W, Lanchbury J S S, Marsh S G E, Hall M A Bodmer J G, Welsh K I. The application of oligonucleotide probes to HLA class II typing of the DRB subregion. Tissue Antigens 1990; 36: 149-55.

12 Gregersen P K, Silver J, Winchester R J. The shared epitope hypothesis. An approach to understanding the molecula genetics of susceptibility to rheumatoid arthritis. Rheumatoid Arthritis 1987; 30: 1205-13.

13 Panayi G S, Wooley P, Batchelor J R. Genetic basis of rheumatoid disease: HLA antigens, disease manifestation and toxic reaction to drugs. $B M \mathcal{F} 1978 ; 2: 1326-8$.

14 Stastny P. Association of the B cell alloantigen DRW4 with rheumatoid arthritis. $N$ Engl f Med 1978; 298: 869-71.

15 Ollier W, Venables P J W, Mumford P A, et al. HLA antigen associations with extraarticular rheumatoid arthritis. Tissue Antigens 1984; 24: 279-91.

16 Olsen N J, Callahan L F, Brooks R H, et al. Association of HLA-DR4 with rheumatoid factor and radiographic severity in rheumatoid arthritis. Am $\mathcal{F}$ Med 1988; 84, 257-64.

17 Calin A, Elswood J, Klouda P T. Destructive arthritis, rheumatoid factor, and HLA-DR4 susceptibility versus severity,

18 Dinant $\mathrm{H} \mathrm{J}$, Muller $\mathrm{W} H$, Van den Berg-Loonen $\mathrm{E} M$ Nijhenhuis L E, Engelfriet C P. HLA-DRW4 in Felty's syndrome [letter] Arthritis Rheum 1980; 23: 1336-7.

19 Campion G, Maddison P J, Goulding N, et al. The Felty syndrome: a case matched study of clinical manifestations and outcome, serologic features and immunogenetic associations. Medicine (Baltimore) 1990; 69: 69-80.

20 Lanchbury J S S, Jaeger E E M, Sansom D M, et al. Strong primary selection for the Dw4 subtype of DR4 accounts for the HLA-DQw7 association with Felty's syndrome. Hum Immunol 1991; 32: 56-64.

21 Gran J T, Husby G, Thorsby E. The association between rheumatoid arthritis and the HLA antigen DR4. Ann Rheum Dis 1983; 42: 292-6.

22 Mottonen $\mathrm{T} T$. Prediction of erosiveness and rate of development of new erosions in early rheumatoid development of new erosions in early

23 Papasteriades C A, Kappou I D, Skopouli F N, Barla M N, Fostiropoulos G A, Moutsopoulos H M. Lack of HLA antigen association in Greek rheumatoid arthritis patients. Rheumatol Int 1985; 5: 201-3.

24 Wordsworth B P, Lanchbury J S S, Sakkas L I, Welsh K I, Panayi G S, Bell J I. HLA-DR4 subtype frequencies in rheumatoid arthritis indicate that DRB1 is the major susceptibility locus within the HLA class II region. Proc Natl Acad Sci USA 1989; 86: 10049-10053.

25 Nepom G T, Byers P, Seyfried C, et al. HLA genes associated with rheumatoid arthritis. Identification of susceptibility alleles using specific oligonucleotide probes. Arthritis Rheum 1989; 32: 15-21.

26 Seglias J, Li E K, Cohen M G, Wong R W S, Potter P K, So A K. Linkage between rheumatoid arthritis susceptibility and the presence of HLA-DR4 and DR $\beta$ allelic third hypervariable region sequences in southern Chinese hypervariable region sequences in south 